\title{
Factors Contributing to Energy Loss in Left Ventricle during Diastolic and Systolic Phases in Elderly Patients.
}

\author{
Shutaro Futami ${ }^{1}$, Joji Ishikawa ${ }^{1}$, Teppei Maeda ${ }^{1}$, Masuyo Kawano $^{1}$, Chieko Sakurayama ${ }^{1}$, \\ and Kazumasa Harada ${ }^{1}$ \\ ${ }^{1}$ Tokyo Metropolitan Geriatric Hospital and Institute of Gerontology
}

July 20, 2020

\begin{abstract}
Background: The change of left ventricular function deteriorated with age because of gradual increases of blood pressure may result in increased energy loss (EL) in left ventricle (LV). The present study investigated EL in LV among hypertensive elderly patients and examined factors contributing to EL. Methods: A single-center retrospective study was performed on elderly hypertensive outpatients (?65 years) who underwent echocardiography ( $\mathrm{N}=105)$. EL in the LV was measured using an vector flow mapping system, and factors affecting peak EL during the early diastolic phase (ED-EL), late diastolic phase (LD-EL), and systolic phase (Sys-EL) were evaluated. Result: Mean age was 79.9 \pm 6.4 years (male 43\%). Mean ED-EL, LD-EL, and Sys-EL were $42.1 \pm 46.7,75.6 \pm 60.2$, and $40.4 \pm 40.2 \mathrm{~mJ} / \mathrm{N} / \mathrm{s}$. In a stepwise regression analysis, the E wave peak velocity of transmitral flow (unstandardized $\mathrm{B}=0.002,95 \% \mathrm{CI} 0.001$ to 0.002 , standardized $\beta=0.547, \mathrm{p}<0.001$ ) and stroke volume in the LV outflow tract (LVOT) ( $\mathrm{B}=0.001,95 \% \mathrm{CI} 0.000$ to $0.001, \beta=0.190, \mathrm{p}=0.034$ ) were identified as factors affecting ED-EL. The factors affecting $\mathrm{LD}-\mathrm{EL}$ were the $\mathrm{E} / \mathrm{A}$ ratio $(\mathrm{B}=-0.122,95 \% \mathrm{CI}-0.180$ to $-0.064, \beta=-0.451, \mathrm{p}<0.001)$ and peak velocity in LVOT (unstandardized $\mathrm{B}=0.001,95 \% \mathrm{CI} 0.0001$ to $0.001, \beta=0.339, \mathrm{p}=0.003)$. The factors influencing Sys-EL were peak velocity in LVOT $(\mathrm{B}=0.001,95 \% \mathrm{CI} 0.001$ to $0.001, \beta=0.619, \mathrm{p}<0.001)$ and the $\mathrm{E} / \mathrm{A}$ ratio $(\mathrm{B}=-0.050,95 \% \mathrm{CI}-0.087$ to $-0.013, \beta=-0.241$, $\mathrm{p}=0.008)$. Conclusion: Peak EL in the LV was higher during diastolic phase than systolic phase among elderly hypertensive patients. Peak EL during each phase was affected by systolic blood flow in LVOT and LV transmitral flow.
\end{abstract}

\section{Introduction}

Aging increases cardiovascular stiffness and structural changes due to cardiomyopathy, hypertension, ischemia, or valvular diseases, resulting in left ventricular (LV) diastolic dysfunction (1) (2) (3) (4). Hypertension is one of the most common problems in the elderly and regarded as one of the most important cardiovascular risk factors (5).

Hypertension in the elderly often progresses to arteriosclerosis and cardiovascular diseases, which changes LV myocardial tissue, such as structural remodeling or impaired function (6). These changes cause LV diastolic dysfunction, which leads to heart failure (HF) with diastolic disfunction despite preserved LV ejection fraction (LVEF) (7). The mortality rates are higher in patients with than in those without LV diastolic dysfunction even when HF is not indicated (8). Therefore, the diagnosis of LV diastolic dysfunction is important. However, the assessment of LV diastolic dysfunction is complicated (9).

Vector flow mapping (VFM) can visualize intraventricular blood flow with velocity vectors $(10,11)$. This new technology is also used to calculate hemodynamic parameters, such as flow energy loss (EL) and shear stress, based on actual measurements. Regarding EL in the LV, three main peaks are observed in one cardiac cycle: in the early diastolic phase (ED), late diastolic phase (LD), and systolic phase (Sys) (12). Previous studies evaluated EL in the LV of healthy adults (29.5 \pm 4.8 years old) (12) and children (13) using VFM. Recent study also showed VFM could be considered as an important supplementary tool to 
quantitatively evaluate the impaired blood flow efficiency and LV diastolic function in patients with various diastolic dysfunction(14).

LV function deteriorates with age because of gradual increases of blood pressure (BP) and the mass of the LV (9). Therefore, the change of LV function caused by aging and increased blood pressure might have the effect to EL in the LV. However, the standard data in the elderly remains unknown and the factors responsible have not yet been identified. The present study was conducted to measure EL in the LV using VFM among elderly patients with hypertension and identify the factors contributing to peak EL during each phase.

\section{Method}

\section{Study population}

A single-center retrospective analysis of echocardiographic data obtained between September 1st 2015 and December 31st 2018 was performed. Data were collected on elderly outpatients with hypertension ([?]65 years) who underwent echocardiography using Profound F 75 Premier (Hitachi Ltd., Tokyo, Japan) (N=105). Hypertension was defined as $\mathrm{BP}[?] 140 / 90 \mathrm{mmHg}$ or the use of antihypertensive medication. Exclusion criteria were as follows: (1) atrial fibrillation at the time of echocardiography, (2) severe valvular diseases, (3) local LV wall motion abnormalities, (4) current decompensated HF, (5) a history of myocardial infarction, and (6) a history of cardiac surgery. The present study was approved by the Ethics Committee of Tokyo Metropolitan Geriatric Hospital (R18-44). Informed consent was not obtained from patients because this was a retrospective analysis of conventional clinical practice data; therefore, information on the opt-out policy was provided on the hospital website.

\section{Data collection}

Data were obtained on clinical history, and venous blood samples at the time of echocardiography. In the hospital, values for eGFR, a measure of glomerular filtration function, were calculated using the Chronic Kidney Disease Epidemiology Collaboration Formula, and serum brain natriuretic peptide (BNP) levels were measured by an enzyme immunoassay (E test TOSOH II (BNP), TOSOH Corporation, Tokyo, Japan) with a coefficient of variation $<15 \%$.

\section{Echocardiography}

Echocardiographic data obtained using the ultrasound device Profound F 75 Premier (Hitachi Ltd, Tokyo, Japan) with a 1-5 MHz sector probe (UST-52105) were analyzed. Echocardiography was performed by experienced sonographers (T.M., M.K., and C.S.) Standard 2D resting echocardiography was conducted in the lateral decubitus position. Conventional echocardiographic data were collected in accordance using the method recommended by the American Society of Echocardiography (9) (15) (16). The modified Simpson's biplane method was used to calculate LVEF and the left atrial volume index (LAVI). Transmitral E and A wave peak velocities were obtained in the apical three-chamber view. Diastolic mitral annular velocities (e' and a') were obtained using Doppler tissue imaging at the septal side of the mitral annulus from the apical four-chamber view. Peak velocities in the LV outflow tract (LVOT), aortic valve, and tricuspid valve were assessed using continuous Doppler imaging.

Cine loop color images from the apical three-chamber view were acquired for use in the VFM analysis. The colored area of this movies is set to include the entire LV area, LV inflow tract, and LVOT during all cardiac cycles. The Doppler area were optimized in order to achieve the highest frame rate. The Nyquist limit set for $2 \mathrm{D}$ color Doppler imaging was $69.3 \mathrm{~cm} / \mathrm{s}$.

\section{Analysis by VFM}

Optimized two-dimensional ultrasound color movies of the apical three-chamber view were transferred to a computer with the VFM software DAS-RS1 (Hitachi Ltd., Tokyo, Japan). Each image of one cardiac cycle was selected using electrocardiography. All aliasing phenomena were checked, and the aliasing area was manually corrected. The LV endocardial boundary was initially traced manually and was then traced 
automatically throughout the remaining frames. The software used calculated intracardiac EL in one cardiac cycle from VFM images.

By echocardiography and electrocardiography, one cardiac cycle was separated into the following three phases: ED, LD and Sys. ED was defined as the phase from the opening of the mitral valve to before the start of atrial contraction. LD was defined as the phase from the start of atrial contraction to the closing of the mitral valve. Peak EL in each phase was collected as a parameter. An example of the VFM analysis in the present study is shown in Figure 1.

\section{Statistical analysis}

Data were shown as means +- standard deviations for continuous variables and percentages for categorical variables. Pearson's correlation coefficients were used to assess relationships between peak EL in ED, LD, and Sys (ED-EL, LD-EL, and Sys-EL, respectively) and age, height, weight, sex, comorbidities, heart rate (HR), BP, laboratory data, and echocardiographic parameters. A stepwise multivariate linear regression was performed to analyze independent variables that correlated with ED-EL, LD-EL, and Sys-EL. All statistical analyses were performed using SPSS software version 20 (IBM Inc., Chicago, IL, USA). A P value less than 0.05 was considered to be significant.

\section{Results}

\section{Patient characteristics}

Patient characteristics are shown in Table $1(\mathrm{~N}=105)$. Mean age was $79.9+-6.4$ years, and $43 \%$ were male. In total, $9 \%$ of patients had a history of paroxysmal atrial fibrillation, but had sinus rhythm at the time of echocardiography. HR and BP were controlled (HR 63.2+-10.9 beats/min, systolic BP $125.8+-17.3 \mathrm{mmHg}$, and diastolic BP 65.4+-11.1 mmHg). The mean serum BNP level was 65.8+-83.2 pg/ml.

Echocardiographic parameters in the present study are shown in Table 2. Patients mostly had preserved LVEF (67.3+-5.6\%) and diastolic dysfunction. The average E wave was $59.3 \mathrm{~cm} / \mathrm{s}, \mathrm{A}$ wave $84.6 \mathrm{~cm} / \mathrm{s}$, and $\mathrm{E} / \mathrm{A}$ ratio 0.72 . Most patients exhibited LA dilatation (LAVI $35.6+-32.4 \mathrm{ml} / \mathrm{m}^{2}$ ).

\section{EL in each phase}

Table 3 shows EL in each phase. ED-EL and LD-EL were higher than Sys-EL, while LD-EL was higher than ED-EL. No significant differences were observed in peak EL between males and females.

\section{Relationships between peak EL during each phase and other parameters (univariate analysis)}

Table 4 shows the correlation coefficients of peak EL to other variables. Figure 2 shows a scatter plot of peak EL during each phase and some variables.

Age, systolic BP, and mean BP did not correlate with peak EL during any phase. Diastolic BP correlated with ED-EL $(\mathrm{r}=-0.251, \mathrm{p}=0.011)$. The LV mass index (LVMI) and LAVI did not correlate with peak EL during any phase.

ED-EL correlated with the indices of diastolic function (E wave peak velocity, A wave peak velocity, E/A, $\mathrm{E} / \mathrm{e}$ ' in the septal side, and $\mathrm{E} / \mathrm{e}^{\prime}$ in the lateral side; $\left.\mathrm{p}<0.05\right)$. LD-EL also correlated with A wave peak velocity $(\mathrm{r}=0.513, \mathrm{p}<0.001)$ and the $\mathrm{E} / \mathrm{A}$ ratio $(\mathrm{r}=-0.358, \mathrm{p}<0.001)$. ED-EL and LD-EL both correlated with the parameters of LVOT (diameter, peak velocity, and the time velocity integral (TVI); $\mathrm{p}<0.05$ ).

Sys-EL correlated with EF $(\mathrm{r}=0.342, \mathrm{p}=0.001)$ and the parameters of LVOT (diameter, peak velocity, and TVI; $\mathrm{p}<0.05)$. It also correlated with the A wave peak velocity $(\mathrm{r}=0.233, \mathrm{p}=0.017)$ and the $\mathrm{E} / \mathrm{A}$ ratio $(\mathrm{r}=-0.240, \mathrm{p}=0.014)$.

\section{Multivariate analysis of relationships between EL and other parameters}

Table 5 shows the correlation coefficients of peak EL during each phase to a number of parameters. Factors contributing to ED-EL were transmitral flow E wave peak velocity and stroke volume in LVOT, while 
those correlated to LD-EL were transmitral flow A wave peak velocity and peak velocity of LVOT. Sys-EL correlated with peak velocity in LVOT and E/A.

\section{Discussion}

The present study assessed peak EL in three major phases of the cardiac cycle in elderly patients with hypertension. The results obtained showed that age and LVMI did not correlate with peak EL in these patients and that the indices of transmitral flow and LVOT parameters were associated with ED-EL, LDEL, and Sys-EL.

Three peaks were observed in EL in the LV cavity of elderly outpatients in the present study, which is consistent with previous findings (12) (13) (14) (17). In the present study, ED-EL and LD-EL were higher than Sys-EL, while LD-EL was higher than ED-EL. These results were in contrast to the findings obtained from children and young healthy volunteers, showing that ED-EL was more likely to be higher than LD-EL (12) (13).

In the present study, a direct correlation was not observed between EL and age; however, EL correlated with the parameters of diastolic function. Diastolic function in the LV decreases with age (1). Aging is associated with cardiovascular stiffness and structural changes due to cardiomyopathy, ischemia, or ventricular dysfunction, resulting in LV diastolic dysfunction. Diastolic function in the LV involves relaxation and compliance. Regarding echocardiographic parameters, reduced LV relaxation generally decreases the E/A ratio and e', leading to an impaired relaxation pattern (9). The JAMP (The Japanese Normal Values for Echocardiographic Measurements Project) study previously reported normal values for echocardiographic parameters in a healthy Japanese population and showed that the age at which $\mathrm{E}$ wave and A wave heights are reversed in a healthy population is approximately 60 years old (18). Patients in the present study were older than 60 years (between 66 and 97 years), and all had hypertension; therefore, LV diastolic dysfunction of varying severities was more prevalent in this group than in children and a young healthy population. The differences observed in EL patterns between the elderly patients in the present study and children or a young healthy population in previous studies were attributed to LV diastolic dysfunction.

In the present study, a higher E wave velocity positively correlated with higher ED-EL. Due to the age of this study group, very few patients had normal diastolic function. Therefore, the higher E wave velocity in this study reflected the severity of LV diastolic dysfunction, resulting in higher ED-EL. Wang et al. previously examined LV diastolic dysfunction and EL in chronic kidney diseases patients with preserved LVEF (LVEF ; 50\%) and sinus rhythm based on blood fluid dynamics using VFM (7). The findings obtained revealed that ED-EL and LD-EL were higher in patients with grade II or III diastolic dysfunction than in those with grade I, and were also higher in patients with grade I diastolic dysfunction than in the control group. The majority of patients in the present study had grade I or II diastolic dysfunction, and the peak diastolic EL pattern observed was similar to that reported previously. Therefore, this suggested that LV diastolic dysfunction caused by aging and hypertension increased ED-EL and LD-EL in the LV cavity.

The proportion of patients with HF and preserved EF (HFpEF) is high in the elderly (16) $(14,19)$. HFpEF often necessitates the evaluation of LV diastolic function. Diastolic dysfunction is assessed by combining various measurements obtained from echocardiography (9); however, this method is complex. The present results and previous findings indicated a relationship between EL in LV and LV diastolic function (14) (20). Therefore, the evaluation of LV EL in each phase, which is simpler than existing methods, has potential as a useful and comprehensive parameter for assessing diastolic function.

Sys-EL correlated with peak velocity in LVOT and the E/A ratio. Peak velocity in LVOT showed a positive correlation. Based on patient backgrounds and exclusion criteria, the patient population examined in the present study had relatively stable cardiac function and none had more than moderate valvular diseases. Therefore, it is considered that morphological changes in LVOT, such as sigmoid septum or hypertensive hypertrophy, which are often observed in elderly patients, affected LVOT parameters. These morphological factors need to be considered in future research. The E/A ratio negatively correlated with Sys-EL. The E/A ratio is a comprehensive index of $\mathrm{LV}$ diastolic function and is used to evaluate left ventricular relaxation 
ability, LV filling pressure, and atrial function (9) (21). In elderly patients, a higher E/A ratio reflects more severe diastolic dysfunction of LV and often accompanies LV systolic dysfunction. Therefore, a higher E/A ratio is associated with lower Sys-EL. The results obtained on the E/A ratio in the present study suggested that diastolic function affected not only diastolic, but also systolic blood flow. Previous studies reported that the incidence of isolated diastolic HF was low and many patients with diastolic HF exhibited subclinical systolic function (22) ((23), which was consistent with the present results on the E/A ratio in Sys.

LVOT TVI and flow velocity positively correlated with ED-EL and LD-EL (Figure 2), suggesting that LVOT blood flow affected LV diastolic function. Blood is viscous and generates frictional heat due to its viscosity at sites at which turbulent flow and vortexes occur. The energy of this frictional heat is called EL in blood flow. EL increases at sites at which large vortexes occur locally and is smaller at those with laminar flow (7) (8) (20). In the present study, LVOT parameters related to vector positively correlated with EL in the LV during ED, LD, and Sys. The morphology of the LVOT affects the formation of LV blood flow. Therefore, the morphology of the LVOT might be associated to intraventricular vector change, not only during Sys, but also ED and LD. Previous studies investigated vortexes in the LV cavity and their contribution to EL (14, 20) (24). The present study did not assess the formation of vortexes. The LVOT diameter did not correlate with EL in this study. Therefore, other factors affecting the morphology of LVOT, such as the angle between LVOT and LV inflow or sigmoid septum, may contribute to EL. In future studies, it is necessary to investigate the effects of these left ventricular outflow tract morphology-related factors on left ventricular blood flow and vortex formation.

\section{Limitations}

The present study was performed at a single center and the number of subjects was small. Accordingly, the results obtained need to be confirmed in a larger number of subjects. Furthermore, it was not possible to evaluate VFM in 3D fields. Blood flow is affected by the structure of the LV. Therefore, evaluations of blood flow in 3D fields will be more precise than those in 2D fields. This is a technical limitation that will be overcome with technological advances in VFM. Another limitation was the severity of diastolic dysfunction. The results of the present study showed diastolic dysfunction may affect peak EL in ED, LD, and Sys. The grading of LV diastolic dysfunction and comparisons of peak EL in each phase may result in more detailed assessments. Therefore, the relationship between EL and the grade of diastolic dysfunction needs to be investigated in more detail in future studies. The present study also did not evaluate vortexes, which need to be assessed in each phase.

\section{Conclusion}

Among elderly patients with hypertension, peak EL in the LV was higher during ED and LD than during Sys, and peak EL in the LV during the three phases was affected by systolic blood flow in LVOT and LV transmitral flow. It also suggested that diastolic dysfunction may be an important factor influencing EL.

\section{Disclosures}

None.

\section{References}

1. Tresch DD, McGough MF. Heart failure with normal systolic function: a common disorder in older people. Journal of the American Geriatrics Society. 1995;43(9):1035-42.

2. Yamamoto K, Masuyama T, Sakata Y, Nishikawa N, Mano T, Yoshida J, et al. Myocardial stiffness is determined by ventricular fibrosis, but not by compensatory or excessive hypertrophy in hypertensive heart. Cardiovascular Research. 2002;55(1):76-82.

3. Borbely A, Velden Jvd, Papp Z, Bronzwaer JGF, Edes I, Stienen GJM, et al. Cardiomyocyte Stiffness in Diastolic Heart Failure. Circulation. 2005;111(6):774-81. 
4. Heerebeek Lv, Borbely A, Niessen HWM, Bronzwaer JGF, Velden Jvd, Stienen GJM, et al. Myocardial Structure and Function Differ in Systolic and Diastolic Heart Failure. Circulation. 2006;113(16):1966-73.

5. Ponikowski P, Voors AA, Anker SD, Bueno H, Cleland JGF, Coats AJS, et al. 2016 ESC Guidelines for the Diagnosis and Treatment of Acute and Chronic Heart Failure. Revista espanola de cardiologia (English ed). 2016;69(12):1167.

6. Pfeffer MA, Shah AM, Borlaug BA. Heart Failure With Preserved Ejection Fraction In Perspective. Circ Res. 2019;124(11):1598-617.

7. Paulus WJ, Tschope C. A novel paradigm for heart failure with preserved ejection fraction: comorbidities drive myocardial dysfunction and remodeling through coronary microvascular endothelial inflammation. Journal of the American College of Cardiology. 2013;62(4):263-71.

8. Redfield MM, Jacobsen SJ, Burnett JC, Jr., Mahoney DW, Bailey KR, Rodeheffer RJ. Burden of systolic and diastolic ventricular dysfunction in the community: appreciating the scope of the heart failure epidemic. Jama. 2003;289(2):194-202.

9. Nagueh SF, Smiseth OA, Appleton CP, Byrd BF, 3rd, Dokainish H, Edvardsen T, et al. Recommendations for the Evaluation of Left Ventricular Diastolic Function by Echocardiography: An Update from the American Society of Echocardiography and the European Association of Cardiovascular Imaging. Journal of the American Society of Echocardiography : official publication of the American Society of Echocardiography. 2016;29(4):277-314.

10. Itatani K, Okada T, Uejima T, Tanaka T, Ono M, Miyaji K, et al. Intraventricular Flow Velocity Vector Visualization Based on the Continuity Equation and Measurements of Vorticity and Wall Shear Stress. Japanese Journal of Applied Physics. 2013;52(7S):07HF16.

11. Sengupta PP, Pedrizzetti G, Kilner PJ, Kheradvar A, Ebbers T, Tonti G, et al. Emerging trends in CV flow visualization. JACC Cardiovascular imaging. 2012;5(3):305-16.

12. Akiyama K, Maeda S, Matsuyama T, Kainuma A, Ishii M, Naito Y, et al. Vector flow mapping analysis of left ventricular energetic performance in healthy adult volunteers. BMC cardiovascular disorders. 2017;17(1):21.

13. Hayashi T, Itatani K, Inuzuka R, Shimizu N, Shindo T, Hirata Y, et al. Dissipative energy loss within the left ventricle detected by vector flow mapping in children: Normal values and effects of age and heart rate. Journal of cardiology. 2015;66(5):403-10.

14. Wang W, Wang Y, Chen X, Yuan L, Bai H. Evaluation of left ventricular diastolic function based on flow energetic parameters in chronic kidney disease with diastolic dysfunction. Echocardiography (Mount Kisco, NY). 2019;36(3):567-76.

15. Picard MH, Adams D, Bierig SM, Dent JM, Douglas PS, Gillam LD, et al. American Society of Echocardiography recommendations for quality echocardiography laboratory operations. Journal of the American Society of Echocardiography : official publication of the American Society of Echocardiography. 2011;24(1):1-10.

16. Lang RM, Badano LP, Mor-Avi V, Afilalo J, Armstrong A, Ernande L, et al. Recommendations for cardiac chamber quantification by echocardiography in adults: an update from the American Society of Echocardiography and the European Association of Cardiovascular Imaging. European heart journal cardiovascular Imaging. 2015;16(3):233-70.

17. Stugaard M, Koriyama H, Katsuki K, Masuda K, Asanuma T, Takeda Y, et al. Energy loss in the left ventricle obtained by vector flow mapping as a new quantitative measure of severity of aortic regurgitation: a combined experimental and clinical study. European Heart Journal - Cardiovascular Imaging. 2015;16(7):723-30. 
18. Daimon M, Watanabe H, Abe Y, Hirata K, Hozumi T, Ishii K, et al. Normal values of echocardiographic parameters in relation to age in a healthy Japanese population: the JAMP study. Circulation journal : official journal of the Japanese Circulation Society. 2008;72(11):1859-66.

19. Stugaard M, Koriyama H, Katsuki K, Masuda K, Asanuma T, Takeda Y, et al. Energy loss in the left ventricle obtained by vector flow mapping as a new quantitative measure of severity of aortic regurgitation: a combined experimental and clinical study. European heart journal cardiovascular Imaging. 2015;16(7):72330 .

20. Cao Y, Sun XY, Zhong M, Li L, Zhang M, Lin MJ, et al. Evaluation of hemodynamics in patients with hypertrophic cardiomyopathy by vector flow mapping: Comparison with healthy subjects. Experimental and therapeutic medicine. 2019;17(6):4379-88.

21. Nagueh SF, Middleton KJ, Kopelen HA, Zoghbi WA, Quinones MA. Doppler tissue imaging: a noninvasive technique for evaluation of left ventricular relaxation and estimation of filling pressures. Journal of the American College of Cardiology. 1997;30(6):1527-33.

22. Nishikage T, Nakai H, Lang RM, Takeuchi M. Subclinical left ventricular longitudinal systolic dysfunction in hypertension with no evidence of heart failure. Circulation journal : official journal of the Japanese Circulation Society. 2008;72(2):189-94.

23. Fukuta H, Little WC. Contribution of systolic and diastolic abnormalities to heart failure with a normal and a reduced ejection fraction. Progress in cardiovascular diseases. 2007;49(4):229-40.

24. Sherrid MV, Kushner J, Yang G, Ro R. Mitral valve coaptation and its relationship to late diastolic flow: A color Doppler and vector flow map echocardiographic study in normal subjects. Echocardiography (Mount Kisco, NY). 2017;34(4):537-48.

\section{Figure Legends}

Figure 1: An example VFM analysis of three phases.

Peak energy loss in the left ventricle in one cardiac cycle was observed in the following three phases: the early-diastolic phase, late-diastolic phase, and systolic phase.

LA, left atrium; LV, left ventricle; Ao, Ascending Aorta.

Figure 2: A scatter plot of peak energy loss during each phase versus variables.

EL, energy loss; ED, early-diastolic phase; TMF, transmitral flow; LVOT, left ventricular outflow tract; TVI, time velocity integral; LD, late-diastolic phase; E/A, peak velocity of the transmitral E wave to that of the A wave.

\section{Hosted file}

Table (Energy loss in LV among the elderly).docx available at https://authorea.com/users/ 344158/articles/470827-factors-contributing-to-energy-loss-in-left-ventricle-duringdiastolic-and-systolic-phases-in-elderly-patients

\section{Hosted file}

Figure (Energy loss in LV among the elderly).pptx available at https://authorea.com/users/ 344158/articles/470827-factors-contributing-to-energy-loss-in-left-ventricle-duringdiastolic-and-systolic-phases-in-elderly-patients 